\title{
Multiverse optimisation based technique for solving economic dispatch in power system
}

\author{
Muhammad Haziq Suhaimi ${ }^{1}$, Ismail Musirin ${ }^{2}$, Muzaiyanah Hidayab ${ }^{3}$, Shahrizal Jelani ${ }^{4}$, \\ Mohd Helmi Mansor ${ }^{5}$ \\ ${ }^{1,2}$ Faculty of Electrical Engineering, Universiti Teknologi MARA, Malaysia \\ ${ }^{3,4}$ Faculty of Engineering, Technology and Built Environment, UCSI University, Malaysia \\ ${ }^{5}$ Department of Electrical and Electronic Engineering, College of Engineering, Universiti Tenaga Nasional, Malaysia
}

\begin{abstract}
Article Info
Article history:

Received Feb 5, 2020

Revised Apr 6, 2020

Accepted Apr 20, 2020

\section{Keywords:}

Economic dispatch

Evolutionary programming

Multiverse optimisation

ABSTRACT

Economic dispatch (ED) is one of the many important components in a power system operation. It is designed to calculate the exact amount of power generation needed to ensure a minimum cost of generation. A power system with multiple generators should be running under an economic condition. The operating cost has to be minimised for any feasible load demand. The increase of power demand is getting higher throughout the year. Economic dispatch is used to schedule and control all output of the fossilfuel or coal-generators to satisfy the system load demand at a minimum cost. This paper presents the Multiverse Optimisation (MVO) for solving the economic dispatch in a power system. The proposed Multiverse optimisation engine developed in this study is implemented on the IEEE 30-Bus Reliability Test System (RTS). It has five generators, all of which are denoted as the control variables for the optimisation process. To reveal the superiority of MVO, a similar process was conducted using Evolutionary Programming (EP). Results from both techniques were compared, and it was revealed that MVO had outperformed EP in terms of reduced cost of generation for the system.
\end{abstract}

Copyright $($ C 2020 Institute of Advanced Engineering and Science. All rights reserved.

\section{Corresponding Author:}

Muzaiyanah Hidayab,

Faculty of Engineering,

Technology and Built Environment,

UCSI University, Kuala Lumpur, Malaysia.

Email: muzaiyanah@ucsiuniversity.edu.my

\section{INTRODUCTION}

Generation of power in a utility always aims for minimum cost of generation; this is termed as Economic Dispatch (ED). ED requires optimisation process, where these optimisation techniques have been in existence for a very long time and can be integrated into the ED system. Previously, many engineers have been utilising the calculative methods to solve ED problems, such as Lambda Iteration and Lagrange Multipliers techniques [1]. In the modern era, engineers and researchers have taken interest in Artificial Intelligence (AI), including that of Genetic Algorithm (GA) technique [2], Particle Swarm Optimisation (PSO) [3], and Firefly Algorithm [4], all of which can be used in many different conditions to obtain better and more appropriate results. However, the issues with these methods are that they are not very accurate and can behave speculatively while taking a longer time to compute, as well as producing random population that will generate the local minimum or maximum value.

Traditional analytics-based strategies neglect to address these types of issues accordingly. In contrast to a portion of the customary calculations, Dynamic Programming (DP) [5] forces no confinements on the idea of bending cost. Consequently, it is often adopted to curb the ED issues with innately nonlinear and intermittent cost bends. This strategy experiences the "scourge of dimensionality" or neighborhood optimality issue. 


\section{LITERATURE REVIEW}

\subsection{Economic dispatch}

Economic dispatch is a generation distribution issue and is characterised as the way toward ascertaining the age unit with the goal that the framework of burden provided is most economical, as per the general inclination requirements. Generally, ED has been adopted since 1920. It was when engineers were stressed over the issue of money related to the dispatching power from generation to consumers. And how to find the most economical division of plant load between the dispatchable generators.

M. Mahmoodi, et al. [6] represented the ED problem with fuel cost, emission, and system failure objectives as a nonlinear multi-objective problem. The future approach of MODE takes an external elitist archive to maintain an algorithm for environmental/economic power transmission non-dominated in MultiObjective Differential Evaluation (MODE).

Z. Li, et al. [7] have developed an algorithm for units with non-smooth fuel cost functions based on Evolutionary Programming (EP) for general ED. The newly developed algorithm is capable of determining global or near-global optimal dispatch solutions. The results show that the proposed EP-based ED algorithm can provide accurate shipper solutions for all types of fuel cost functions within an appropriate time frame.

Engineers still continue searching for the best answer to spare the activity expenses of intensity ages. Generators must have good productivity level and economic. The goal of comprehending the ED issue is to have control over the yield output of the generators so as to limit the total out-of-pocket cost while supporting the heap power request and different requirements. The ED objective for optimal load dispatch is to reduce the fuel cost of thermal generators while satisfying some of the limits. The function is given by:

$$
C=\sum_{i=1}^{N G}\left(a_{i} P_{g i}^{2}+b_{i} P_{g i}+c_{i}\right)
$$

where, $a_{i}, b_{i}$, and $c_{i}$ are the fuel-cost coefficients; and $P_{g i}$ is the power output for the $i^{t h}$ generator among the total committed generators. The overall fuel cost has to be reduced through the following constraints:

\subsubsection{Energy balance constraint}

The overall generation by all generators should be equal to the sum of whole power demand, $P_{d}$ and the system's real power loss, $P_{L}$.

$$
\sum_{i=1}^{N G} P_{g i}-P_{d}=P_{L}
$$

The power loss is calculated by using $B$ coefficients and unit power output:

$$
P_{L}=\sum_{i=1}^{N G} \sum_{j=1}^{N G} P_{i} B_{i j} P_{j}+\sum_{i=1}^{N G} B_{0 i} P_{i}+B_{00}
$$

\subsubsection{Operatimg limits constraint}

The dispatchable generators must be ensured operating within the allowable limits in order to avoid instability in their operation.

\subsection{Evolutionary progamming}

EP was first developed by Fogel and family, where it was proposed to be used for the advancement of limited state machines to illuminate expected undertakings [8]. From that point onwards, alterations, improvement, and executions have been proposed and explored. The processes of original EP are as shown in Figure 1. EP has been utilised to treat genuine esteemed item factors or some other plausible information structures. Change is frequently actualised by including an arbitrary number or a vector from a specific dispersion [e.g., a Gaussian adaptation on the account of old-style EP (CEP)] to a parent. The variety level of the Gaussian transformation is constrained by its standard deviation, which is otherwise called a methodology parameter in developmental inquiry [9-11]. In the self-adjustment plan of EP, this parameter is not prefixed. It is assumed to have been developed alongside the goal factors. Analyses with self-versatile EP have demonstrated proficient intermingling to quality arrangements $[12,13]$.

\subsection{Multiverse optimisation (MVO)}

The MVO technique was first proposed by Seyedali Mirjalili [14-16]. It was invented to simulate the behaviour of white holes, worm holes, and black holes. These holes are as shown in Figure 2. A white hole did not actually exist in the universe, but some astronomical scientists have assumed that the Big Bang can act as a white hole that resulted in the Big Bang occurrence. In a multiverse theory, it is assumed that the parallels had collided with one another when the big bang transpired. A black hole that has the opposite role 
of a white hole had absorbed all of the objects and planets around them with their force of gravity [17-19]. Wormholes are holes that are interconnected with the different corners of a universe. In the multiverse theory, wormholes act as a time or space travel path, where objects are able to move instantly from one part to any other part of a universe [20-23].

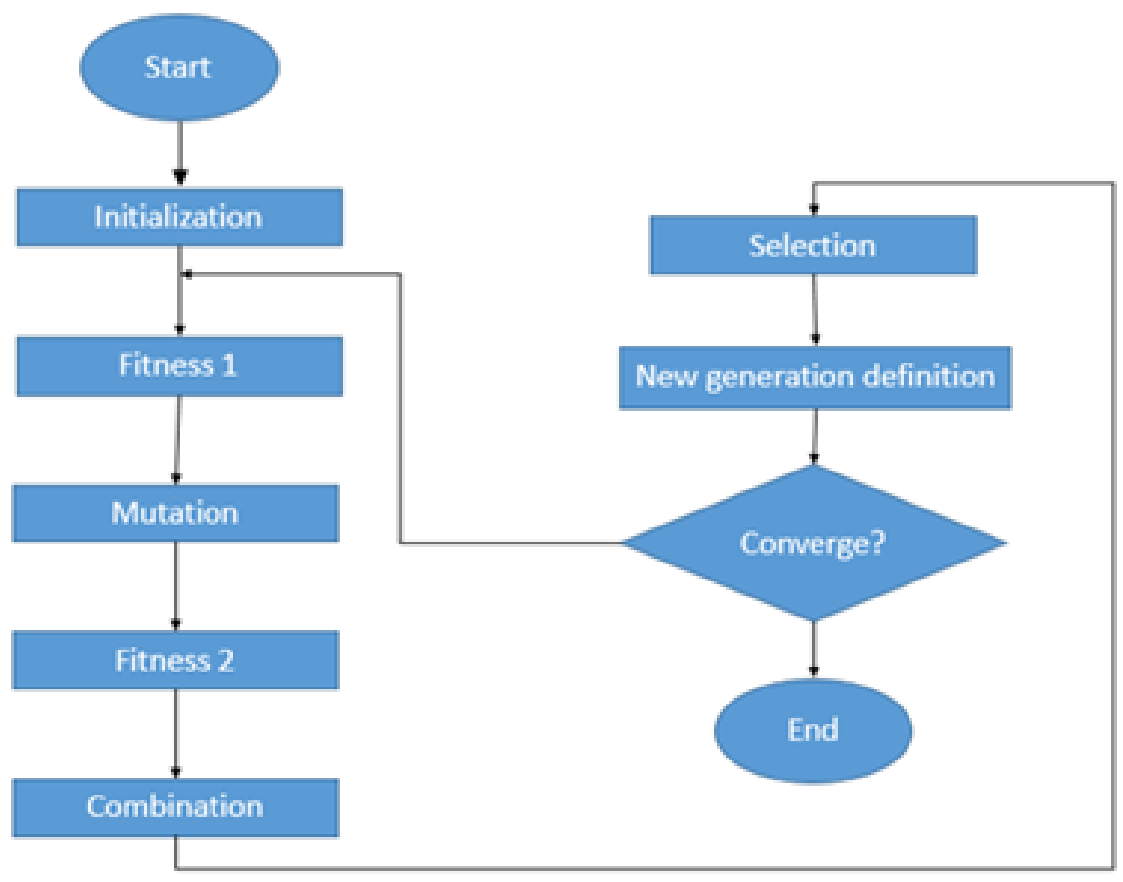

Figure 1. Evolutionary programming algorithm
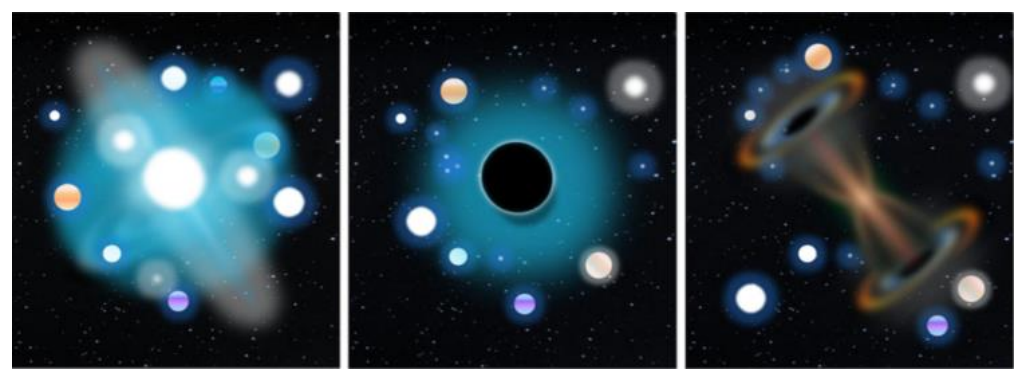

Figure 2. Image of white hole, black hole and worm hole [14]

As previously mentioned, a populace-based calculation separates the pursuit procedure into two stages: investigation versus abuse. The researcher had adopted the idea of white opening and dark gap so as to investigate the search space through the exploitation of MVO. Conversely, the wormholes helped MVO in abusing the inquiry spaces. It is expected that every arrangement is similar to a universe and every factor in the arrangement is an article in that universe. In addition, each arrangement had been allocated a swelling rate, which is relative to comparing the wellness work estimation of the arrangement. Moreover, the term "time" was utilised in this paper rather than the term "cycle", since it is a typical term in multi-stanza hypothesis and cosmology [24-26]. During the optimisation process, the rules to be applied towards the universe of the MVO are:

a) Increase of inflation rate will increase the probability of a white hole occurrence;

b) Increase of inflation rate will decrease the probability of a black hole occurrence;

c) Universe with a higher inflation rate will tend to send objects through the white hole;

d) Universe with a lower inflation rate will tend to send objects through the black hole; and

e) All objects in all of the universes may face a random movement towards the best universe that it would fit through the wormhole regardless the inflation rate.

Multiverse optimisation based technique for solving economic dispatch ... (Muhammad Haziq Suhaimi) 
Two main coefficients for MVO are:

1. Wormhole Existence Probability

$W E P=\min +l \times\left(\frac{\max -\min }{L}\right)$

2. Traveling Distance Rate

$$
T D R=\left(1-\frac{l^{\frac{1}{p}}}{L^{\frac{1}{p}}}\right)
$$

MVO algorithm simulates the concept of the universe setting up the number of universes that are desirable, comprising data functions and number of maximum iterations. MVO algorithm is illustrated in Figure 3. MVO will produce the universe inflation, sort it, and normalise the data. This will automatically update the position of each universe and calculate the value of WEP and TDR of every universe. The Roulette Wheel Selection (RWS) algorithm is used to create white holes as indices, depending on the WEP and TDR values. Subsequently, the optimisation engine will update the convergence curve and create the convergence graph.

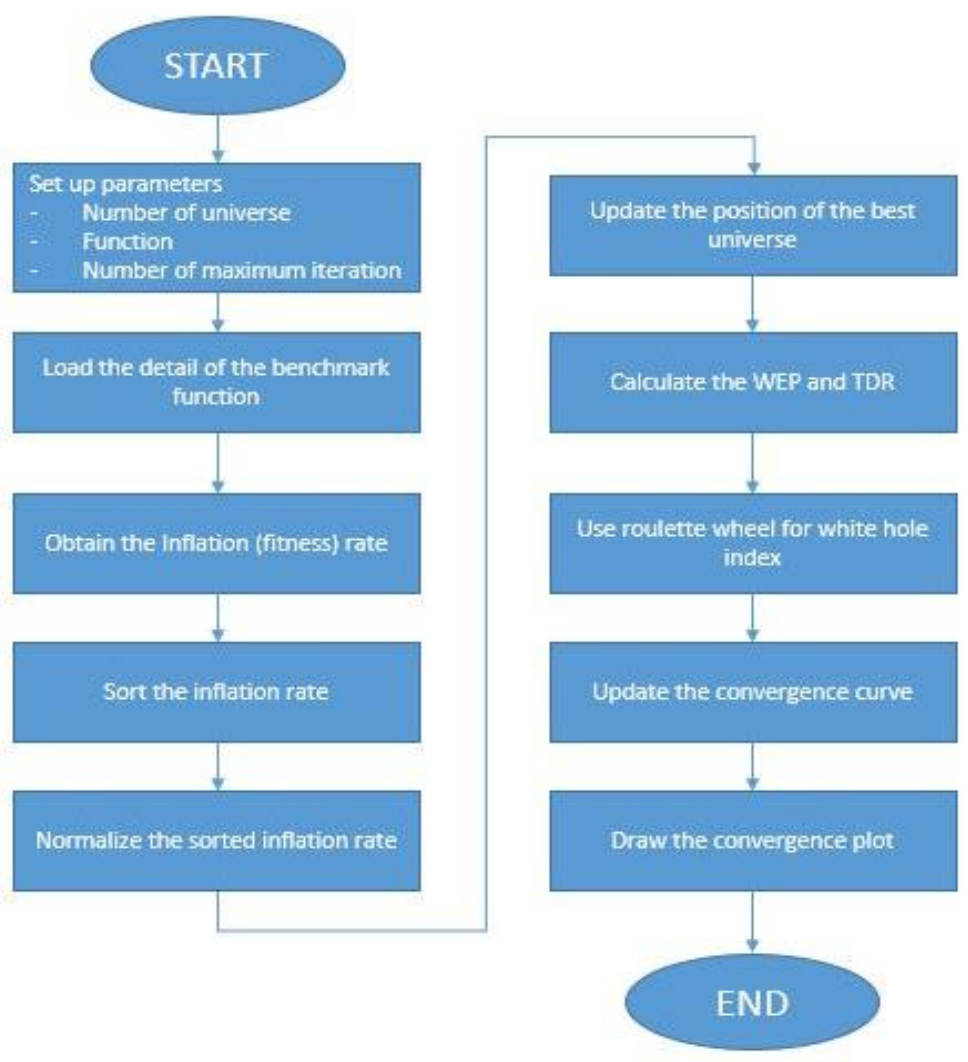

Figure 3. MVO processes

\section{RESULTS AND DISCUSSION}

In this paper, the MVO and EP were used to determine the optimal generation of the IEEE 30-Bus RTS. The ED program was developed in MATLAB(R2018a), where the population size and the maximum number of iteration were 60 and 500, respectively.

\subsection{Case 1}

The first case is the generators had run to cater $100 \%$ of power demand (PD), which is $800 \mathrm{MW}$. Table 1 shows the ED result via MVO: the power generation of each generator, as well as their power losses and total 
generation cost. From the table above-mentioned, the power generated by Gen 1, Gen 2, Gen 3, Gen 4, Gen 5, and Gen 6 are 32.61 MW, 14.48 MW, 141.56 MW, 136.04 MW, 257.59 MW, and 243.06 MW, respectively. These are the values to achieve the total generation cost of $41896.63 \$ / \mathrm{h}$. On the other hand, Table 2 demonstrates the ED result via EP. It shows that the power generated by Gen 1, Gen 2, Gen 3, Gen 4, Gen 5, and Gen 6 at MVO values of 28.06 MW, 17.65 MW, 157.60 MW, 150.62 MW, 241.08 MW, and 229.70 MW, respectively. These values also serve to achieve the total generation cost of $43620.88 \$ / \mathrm{h}$. Apparently, with the implementation of MVO, the total generation cost worth $41896.63 \$ / \mathrm{h}$ can be minimised, as compared to that using EP, which can only minimise it to $43620.88 \$ / \mathrm{h}$. This shows that there was a $3.95 \%$ cost reduction by using MVO compared to using EP.

Table 1. ED result via MVO for $\mathrm{PD}=800 \mathrm{MW}$

\begin{tabular}{cc}
\hline Unit & Value \\
\hline Gen 1 (MW) & 32.61 \\
Gen 2 (MW) & 14.48 \\
Gen 3 (MW) & 141.56 \\
Gen 4 (MW) & 136.04 \\
Gen 5 (MW) & 257.59 \\
Gen 6 (MW) & 243.06 \\
Power Loss (MW) & 25.33 \\
Total Generation & 41896.63 \\
Cost (\$/h) & \\
\hline
\end{tabular}

Table 2. ED result via EP for PD $=800 \mathrm{MW}$

\begin{tabular}{cc}
\hline Unit & Value \\
\hline Gen 1 (MW) & 28.06 \\
Gen 2 (MW) & 17.65 \\
Gen 3 (MW) & 157.60 \\
Gen 4 (MW) & 150.62 \\
Gen 5 (MW) & 241.08 \\
Gen 6 (MW) & 229.70 \\
Power Loss (MW) & 24.70 \\
Total Generation & 43620.88 \\
Cost (\$/h) & \\
\hline
\end{tabular}

\subsection{Case 2}

The second case is when the PD was $600 \mathrm{MW}$, operating $75 \%$ of the maximum power demand. The result of ED via MVO: generators output, power loss, and total generation cost are shown in Table 3 . In this table, values of 23.91 MW, 10, 95.54 MW, 100.84 MW, 202.80 MW, and 181.15 MW were obtained for the power generated by Gen 1, Gen 2, Gen 3, Gen 4, Gen 5, and Gen 6, respectively. These are the values for achieving $32094.68 \$ / \mathrm{h}$ of the total cost of generation. On the other hand, Table 4 of ED results via EP portrays values of $20.70 \mathrm{MW}, 10.00 \mathrm{MW}, 110.65 \mathrm{MW}, 112.23 \mathrm{MW}, 188.00 \mathrm{MW}$, and 172.31 MW for the power generated by Gen 1, Gen 2, Gen 3, Gen 4, Gen 5, and Gen 6, respectively. This resulted in a total generation cost of $33066.35 \$ / \mathrm{h}$. Consequently, there can be a minimum reduction in the total generation cost of $32094.68 \$ / \mathrm{h}$ by implementing MVO, in comparison to EP which only succeeded in minimising the numbers to $33066.35 \$ / \mathrm{h}$. This shows that the cost of using MVO is $2.94 \%$ lower than that using EP.

Table 3. ED result via MVO for $\mathrm{PD}=600 \mathrm{MW}$

\begin{tabular}{cc}
\hline Unit & Value \\
\hline Gen 1 (MW) & 23.91 \\
Gen 2 (MW) & 10.00 \\
Gen 3 (MW) & 95.54 \\
Gen 4 (MW) & 100.84 \\
Gen 5 (MW) & 202.80 \\
Gen 6 (MW) & 181.15 \\
Power Loss (MW) & 14.24 \\
Total Generation & 32094.68 \\
Cost (\$/h) & \\
\hline
\end{tabular}

Table 4. ED result via EP for $\mathrm{PD}=600 \mathrm{MW}$

\begin{tabular}{cc}
\hline Unit & Value \\
\hline Gen 1 (MW) & 20.70 \\
Gen 2 (MW) & 10.00 \\
Gen 3 (MW) & 110.65 \\
Gen 4 (MW) & 112.23 \\
Gen 5 (MW) & 188.00 \\
Gen 6 (MW) & 172.31 \\
Power Loss (MW) & 13.88 \\
Total Generation & 33066.35 \\
Cost (\$/h) & \\
\hline
\end{tabular}

\subsection{Case 3}

The third case is when the PD was 400 MW. Table 5 shows the ED result via MVO of power loss and total generation cost per generator output. This table includes the power generated from Gen 1, Gen 2, Gen 3, Gen 4, Gen 5, and Gen 6, which had values of 14.84 MW, 10.47.92 MW, 63.77 MW, 144.89 MW, and 125.00 MW, respectively. These are the values to achieve a total generation cost of $22952.83 \$ / \mathrm{h}$. While Table 6 of ED result via EP shows the power generated by Gen 1, Gen 2, Gen 3, Gen 4, Gen 5, and Gen 6 were 12.66 MW, 10.02 MW, 58.54 MW, 69.92 MW, 130.10 MW, and 125.00 MW, respectively. This resulted in a total generation cost of $23400.84 \$ / \mathrm{h}$. As a result, the total generation cost was reduced to a minimum of $22952.83 \$ / \mathrm{h}$ through the implementation of MVO, in comparison to the EP which only succeeded in minimising it to $23400.84 \$ / \mathrm{h}$. This show that the total generation cost produced using MVO is $1.91 \%$ lower than that using ED. 


\begin{tabular}{|c|c|}
\hline Unit & MVO \\
\hline Gen 1 (MW) & 14.83 \\
\hline Gen 2 (MW) & 10.00 \\
\hline Gen 3 (MW) & 47.92 \\
\hline Gen 4 (MW) & 63.77 \\
\hline Gen 5 (MW) & 144.89 \\
\hline Gen 6 (MW) & 125.00 \\
\hline Power Loss (MW) & 6.41 \\
\hline $\begin{array}{l}\text { Total Generation } \\
\text { Cost }(\$ / \mathrm{h})\end{array}$ & 22592.83 \\
\hline
\end{tabular}

\begin{tabular}{cc} 
Table 6. ED result via EP for PD $=400$ \\
\hline Unit & MVO \\
\hline Gen 1 (MW) & 12.66 \\
Gen 2 (MW) & 10.02 \\
Gen 3 (MW) & 58.54 \\
Gen 4 (MW) & 69.92 \\
Gen 5 (MW) & 130.10 \\
Gen 6 (MW) & 125.00 \\
Power Loss (MW) & 6.24 \\
Total Generation & 23400.84 \\
Cost (\$/h) & \\
\hline
\end{tabular}

\section{CONCLUSIONS}

The MVO technique is programmed to imitate the behaviour of white holes, black holes, and worm holes by creating the best universe out of all the variables. This work proposes the Multiverse Optimisation for solving the economic power dispatch (ED) problem in comparison to utilising the Evolutionary Programming. The test was conducted in three different cases, which are $100 \%$ power demand, $75 \%$ power demand and $50 \%$ power demand.

Out of all three cases in the above, the adoption of MVO technique has proven to be more reliable and superior than that using EP in solving ED problems, where all of the results taken from the MVO showed that the total generation cost is lower than the results taken from EP, thus achieving the main objectives of this research. For future studies, MVO can be utilised to solve similar problems in a power system, which may require minor modifications on the developed optimisation engine.

\section{ACKNOWLEDGEMENT}

The authors would like to acknowledge the UCSI University for the financial support given for this project. This research is supported by the UCSI University under the Centre of Excellence for Research, Value Innovation, and Entrepreneurship (CERVIE).

\section{REFERENCES}

[1] J. A. Muckstadt and S. A. Koenig, "An Application of Lagrangian Relaxation to Scheduling in Power-Generation Systems," Operations research, vol. 25, no. 3, pp. 387-403, 1977.

[2] S. A. Shaaya, et al., "Effect of optimal multi-DG siting and sizing in transmission system using hybrid optimization technique for voltage control," Indonesian Journal of Electrical Engineering and Computer Science (IJEECS), vol. 17, no. 2, pp. 646-653, 2019.

[3] R. Habachi, et al., "Resolution of economic dispatch problem of the moroccan network using crow search algorithm," Indones. J. Electr. Eng. Comput. Sci., vol. 13, no. 1, pp. 347-353, 2019.

[4] S. C. Mohd Nasir, et al., "Multistage artificial immune system for static VAR compensator planning," Indonesian Journal of Electrical Engineering and Computer Science (IJEECS), vol. 14, no. 1, pp. 346-352, 2019

[5] Y. Xu, et al., "Distributed dynamic programming-based approach for economic dispatch in smart grids," IEEE Trans. Ind. Informatics, vol. 11, no. 1, pp. 166-175, 2014.

[6] M. Mahmoodi, et al., "Economic dispatch of a hybrid microgrid with distributed energy storage," IEEE Trans. Smart Grid, vol. 6, no. 6, pp. 2607-2614, 2015.

[7] Z. Li, et al., "Sufficient Conditions for Exact Relaxation of Complementarity Constraints for Storage-Concerned Economic Dispatch,” IEEE Trans. Power Syst., vol. 31, no. 2, pp. 1653-1654, 2015.

[8] D. B. Fogel and L. J. Fogel, "An introduction to evolutionary programming," European Conference on Artificial Evolution. Springer, pp. 21-33, 1995.

[9] S. Surender Reddy, et al., "Real-Time Economic Dispatch Considering Renewable Power Generation Variability and Uncertainty over Scheduling Period," IEEE Syst. J., vol. 9, no. 4, pp. 1440-1451, 2014.

[10] M. S. P. Subathra, et al., "A hybrid with cross-entropy method and sequential quadratic programming to solve economic load dispatch problem," IEEE Syst. J., vol. 9, no. 3, pp. 1031-1044, 2014.

[11] Z. Li, et al., "Combined heat and power dispatch considering pipeline energy storage of district heating network," IEEE Trans. Sustain. Energy, vol. 7, no. 1, pp. 12-22, 2015.

[12] M. H. Mansor, et al., "Immune Log-Normal Evolutionary Programming (ILNEP) for solving economic dispatch problem with prohibited operating zones," 2017 4th Int. Conf. Ind. Eng. Appl. ICIEA, pp. 163-167, 2017.

[13] Y. del Valle, et al., "Particle swarm optimization: Basic concepts, variants and applications in power systems," IEEE Transactions on Evolutionary Computation, vol. 12, no. 2, pp. 171-195, 2008.

[14] G. I. Sayed, et al., "Quantum multiverse optimization algorithm for optimization problems," Neural Computing and Applications, pp. 1-18, 2017.

[15] S. Mirjalili, et al., "Multi-Verse Optimizer: a nature-inspired algorithm for global optimization," Neural Comput. Appl., vol. 27, no. 2, pp. 495-513, 2016. 
[16] X. Liu, M. Ding, J. Han, P. Han, and Y. Peng, "Dynamic economic dispatch for microgrids including battery energy storage," in 2nd International Symposium on Power Electronics for Distributed Generation Systems, PEDG 2010, pp. 914-917, 2010.

[17] D. wei Gong, et al., "Environmental/economic power dispatch using a hybrid multi-objective optimization algorithm," Int. J. Electr. Power Energy Syst., vol. 32, no. 6, pp.607-614, 2010.

[18] M. Ghasemi, et al., "A differential evolution particle swarm optimizer for various types of multi-area economic dispatch problems," Energy, vol. 107, pp.182-195, 2016.

[19] Á. Lorca and X. A. Sun, "Adaptive Robust Optimization With Dynamic Uncertainty Sets for Multi-Period Economic Dispatch Under Significant Wind," IEEE Trans. Power Syst., vol. 30, no. 4, pp. 1702-1713, 2014.

[20] H. Gangammanavar, et al., "Stochastic Optimization of Sub-Hourly Economic Dispatch with Wind Energy," IEEE Trans. Power Syst., vol. 31, no. 2, pp. 949-959, 2015.

[21] Q. Wu, "Cauchy mutation for decision-making variable of Gaussian particle swarm optimization applied to parameters selection of SVM," Expert Syst. Appl., vol. 38, no. 5, pp.4929-4934, 2011.

[22] P. G. Espejo, et al., "A survey on the application of genetic programming to classification," IEEE Transactions on Systems, Man and Cybernetics Part C: Applications and Reviews, vol. 40, no. 2, pp.121-144, 2009.

[23] T. Apostolopoulos and A. Vlachos, "Application of the Firefly Algorithm for Solving the Economic Emissions Load Dispatch Problem," Int. J. Comb., 2010.

[24] Bakirtzis, A., Petridis, V. and Kazarlis, S., "Genetic algorithm solution to the economic dispatch problem," IEE proceedings-generation, transmission and distribution, vol. 141, no. 4, pp.377-382, 1994.

[25] S. Gholizadeh, et al., "Improved black hole and multiverse algorithms for discrete sizing optimization of planar structures," Engineering Optimization, vol. 51, no. 10, pp. 1645-1667, 2019.

[26] Noor Azlan Adnan, et al., "Multi-Objective Evolutionary Programming for Solving Economic Dispatch Problem," Int. J. Adv. Trends Comput. Sci. Eng., vol. 8, no. 1.6, pp. 296 - 302, 2019. 